\title{
VLSI Partitioning of a 2-Gs/s Digital Spectrometer
}

\author{
Brian Von Herzen
}

\begin{abstract}
A digital correlating spectrometer is described for radio astronomy based on a custom GaAs digitizer and a custom micropipelined CMOS correlator. The digitizer quantizes at 2 gigasamples per second (Gs/s) and 2-b resolution. A GaAs demultiplexer distributes the data into eight parallel streams of $250 \mathrm{Ms} / \mathrm{s}$ each. The CMOS correlator operates at $250 \mathrm{Ms} / \mathrm{s}$ using $20 \mathrm{~mW}$ per correlator lag. The correlator output is processed on a host microcomputer to create a 1-GHz spectrum of the input signal that can be displayed interactively. An $8 \times 9$ - $\mathrm{mm}$ chip is being developed in a $2-\mu \mathrm{m}$ process that contains 320 correlator lags. The design is partitioned into GaAs and CMOS components according to the required throughput at each stage of the system. The fastest signals $(2 \mathrm{GHz})$ are kept on the chip level to minimize delay, crosstalk, system noise, and power consumption. Moderate-speed signals $(250 \mathrm{MHz})$ are driven by GaAs components. CMOS components are used where highspeed outputs are not required. A strong synergy between the correlator architecture and micropipelined CMOS technology improves the performance by an order of magnitude compared to existing designs. Preliminary correlator chips have been built and tested at $250 \mathrm{Ms}$ /s; final chips are under design.
\end{abstract}

\section{INTRODUCTION}

$\mathrm{O}$ NE OF THE major challenges in developing a microelectronic VLSI system is to successfully partition the design into separate components. Designs must satisfy simultaneous constraints on area, power, pins, throughput, and noise. This paper examines such a constrained design: a digital correlating spectrometer optimized for radio astronomy. These spectrometers are attractive because of potentially low cost, small size, and high reliability relative to other spectrometers [1]. The correlating spectrometer is partitioned into GaAs and CMOS components to minimize high-speed signals on the circuit board and to eliminate high-speed outputs from the CMOS chips. The characteristics of micropipelined CMOS technology are reviewed, followed by a description of the spectrometer architecture. The CMOS correlator subsystem is examined in detail with a performance comparison to existing correlators.

\section{Micropipelined CMOS Technology}

The CMOS chips in the spectrometer use micropipelining [2], which refers to small and simple pipeline stages placed in series. Each stage might have only one or two

Manuscript received September 20, 1990; revised January 10, 1991. This work was supported by the National Science Foundation through Grant AST 891-2705 to the Caltech Submillimeter Observatory.

The author is with Caltech Submillimeter Observatory, Hilo, HI 96720 .

IEEE Log Number 9143193.

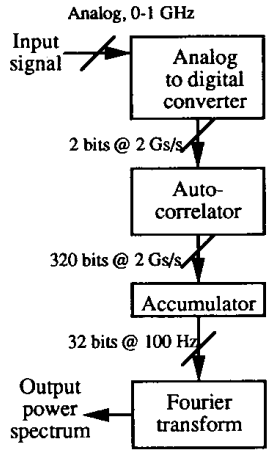

Fig. 1. Digital correlating spectrometer.

logic gates between registers, maximizing the throughput of the circuit. These stages can use asynchronous or synchronous signaling [2].

Micropipelined CMOS circuits can accept input signals of several hundred megahertz if driven by external nonCMOS devices. Micropipelined systems can keep up with these data rates if the maximum latency between stages is only one or two gates [3], and if nearest-neighbor communication is used between functional blocks to minimize capacitance (no global wires driven by CMOS transistors). System latency does increase for micropipelined systems, but many signal processing systems such as the digital spectrometer depend much more on throughput than on latency. Output signals in CMOS are usually restricted to tens of megahertz, due to limited pin-driving capability. These qualities make micropipelined CMOS attractive in architectures requiring high input bandwidths and circuit density but with low output bandwidths. The correlator subsystem of the digital spectrometer matches these characteristics.

\section{Spectrometer Architecture}

A correlating spectrometer computes a power spectrum from the Fourier transform of the autocorrelation of a signal [4]. Fig. 1 shows a block diagram of the signal processing involved. A $1-\mathrm{GHz}$ analog signal is digitized at $2 \mathrm{~b}$ and 2 gigasamples per second (Gs/s). For weak astronomical signals, a 2-b quantizer is nearly as sensitive as an ideal quantizer with many bits. A 2-b quantizer provides $87 \%$ of the ideal sensitivity for $0-\mathrm{dB}$ signals with Gaussian noise [5]. 


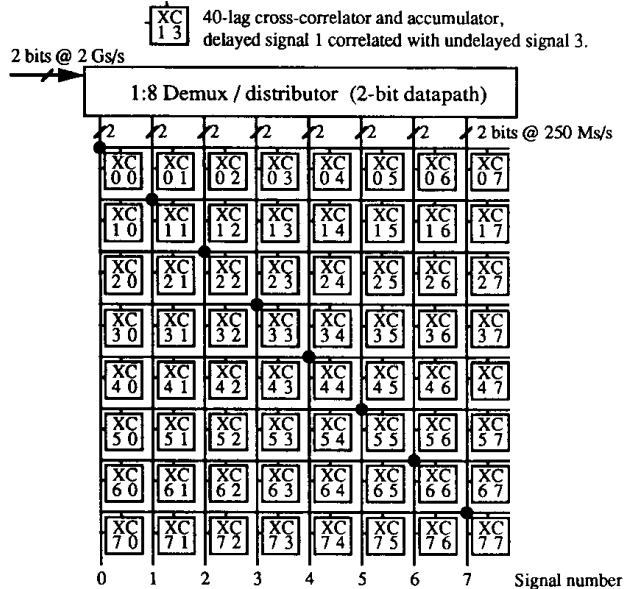

Fig. 2. Correlator architecture.

The correlator takes the digitized signal $s(t)$ and computes the autocorrelation $\sigma(\tau)$ :

$$
\sigma(\tau)=\sum_{t} s(t) s(t-\tau)
$$

Each discrete value of $\tau$ corresponds to a single correlator "lag." A correlator with 320 lags will produce a power spectrum with 320 channels, neglecting windowing effects. Ripple counters accumulate the outputs of the correlator. Since the integration time is long, the bandwidth out of the counters is reduced to a few hundred hertz. If the correlator and accumulator are integrated onto a single chip, the high-frequency outputs are eliminated. This integration permits the CMOS chip to run at $250 \mathrm{MHz}$ without having to generate output signals at $250 \mathrm{MHz}$.

A Fourier transform converts the accumulated autocorrelation function into a power spectrum. This computation can be done in software since the correlator produces 320 data points every few seconds.

\section{Description of the Correlator AND ACCUMULATOR}

Fig. 2 shows the correlator architecture in greater detail. The CMOS correlator chips cannot operate at the full $2-\mathrm{Gs} / \mathrm{s}$ bandwidth so the data are split into eight parallel streams and each data stream is cross-correlated with all the others. Since there are eight data streams, we need 64 cross-correlators to correlate all the data.

The correlator system is partitioned into eight correlator chips, each chip containing eight 40-lag cross-correlators. The host computer reconstructs the autocorrelation by adding together lags of equal time offsets. In Fig. 2 the data paths are numbered $0-7$ and the correlator elements are numbered 00 through 07,10 through $17, \cdots$, and 70 through 77 for each pair of inputs. Correlators 70 and 07 are not redundant because the inputs are not symmetric. Correlator 00 computes lags with time offsets $0,8,16, \cdots$, and 312. Correlator 07 computes lags $7,15,23, \cdots$, and 319. Correlator 70 computes lags $-7,1,9,17, \cdots$, and
305. Correlator 77 computes lags $0,8,16, \cdots$, and 312 . Note that correlators 00 and 77 compute the same time offsets using different data. These lags are added together on the host computer to reconstruct the original autocorrelation function. In this way, all lags from 0 through 312 are computed, with some redundant lags from -7 to -1 and some extra lags from 313 to 319 .

Each cross-correlator has inputs for delayed and undelayed data (Fig. 3). The $Z^{-1}$ symbol refers to unit delay elements, which retard the data by one sample for each correlator lag. A simple dynamic shift register suffices for the delay elements. Each unit delay uses a single pipeline stage.

For each correlator lag, the delayed sample is multiplied by the undelayed sample using a 2-b $\times 2-b$ multiplier. The product is offset and rounded to produce a positive 3 -b result with $<1 \%$ sensitivity degradation [5]. The product is accumulated in a 4-b adder, and the carry output is fed into a ripple-counter chain that integrates the correlation function. A 32-b counter requires $16 \mathrm{~s}$ to overflow in the worst case at $250 \mathrm{Ms} / \mathrm{s}$. Longer integrations can be summed on the host computer. After the integration period, the counters are frozen and the results are sent to the host. The output interface of the correlator chip looks like a standard byte-wide ROM.

\section{Mapping the Correlator into Micropipelined CMOS TECHNOLOGY}

The functional blocks of the correlator and accumulator match extremely well to the capabilities of micropipelined CMOS. High density and throughput are critical for the correlator circuits, and for the first few stages of the accumulator. The output of the accumulator is very slow, and this matches the slower output rates for CMOS devices. As a result, the CMOS correlator runs at ECL speeds of $250 \mathrm{Ms} / \mathrm{s}$ yet still has the density of CMOS.

Power dissipation requires special attention for these chips. Each 320-lag correlator chip is expected to produce over $6 \mathrm{~W}$ of heat based on test chips and SPICE simulations. Aluminum heat sinks are needed on the ceramic PGA packages to dissipate the heat.

Fig. 4 shows a single pipelined correlator lag. The 3-b product (MSB, MID, LSB) is computed in the first two pipeline stages, followed by a 4-b accumulation. Note that there are at most two logic gates between pipeline stages, which results in a high throughput and latency for the correlator. This approach can double the area of a logic circuit, but more than doubles the speed of the circuit compared to a combinatorial approach. The maximum delay goes from nine gates to two gates as the micropipeline registers are placed in the circuit.

The gates use complementary pass-transistor logic [6], which offers fast xOR gates with the same area as NAND and NOR gates. The data path of the correlator uses XOR gates extensively, so complementary pass-transistor logic is particularly attractive. 


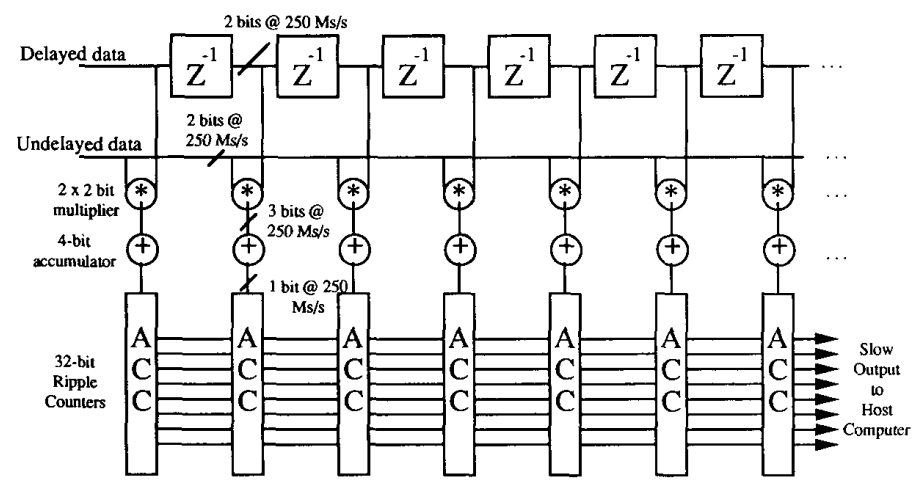

Fig. 3. CMOS correlator chip structure.

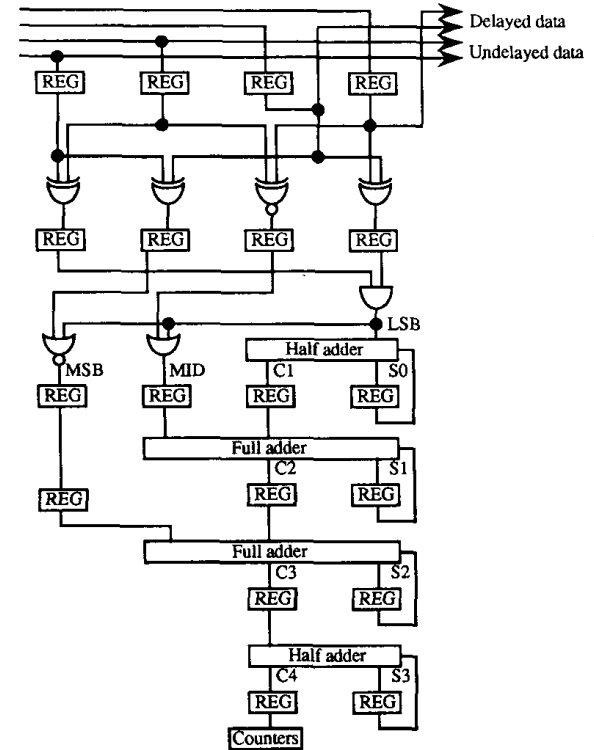

Fig. 4. Logic for a single correlator lag. MSB, MID, and LSB refer to the 3-b product. $S 0-S 3$ hold the 4-b sum. $C 4$ is the carry output from the correlator adder. At most, two logic gates lie between each register stage.

\section{CMOS Correlator Floor Plan}

A critical aspect of the CMOS layout is to minimize the parasitic loading on internal nodes of the correlator chip. Fortunately, the correlator can be laid out exclusively with nearest-neighbor communications between cells. All global clocks and input signals are driven from external GaAs FET's or bipolar devices. This approach eliminates large parasitic capacitances caused by internal global wires.

Fig. 3 shows the basic layout structure of the correlator. The nearest-neighbor communication minimizes the $R C$ time constants associated with the signal wires. The undelayed data lines are driven externally. The layout of the correlator chip is shown in Fig. 5. Twelve lags of the correlator fit onto a single tiny chip fabricated through the MOSIS prototype fabrication service [7]. The chip

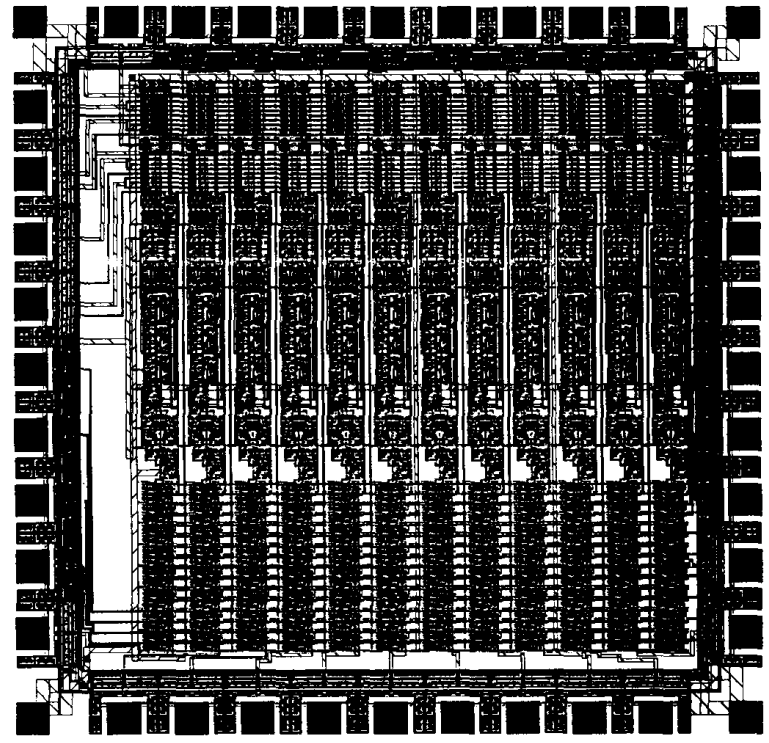

Fig. 5. CMOS correlator with 12 lags and 16-b accumulators on a MOSIS tiny chip.

measures $2.2 \mathrm{~mm}$ on each side. Roughly 320 correlator lags fit onto a chip measuring $8 \times 9 \mathrm{~mm}$.

\section{GaAs Digitizer}

The fastest part of the spectrometer is the A/D subsystem, which consists of a gallium-arsenide flash converter coupled to a $1: 8$ demultiplexer/distributor for the CMOS correlator. The data path from the A/D must run at 2 Gs/s. The distributor reduces the bandwidth to $250 \mathrm{Ms} / \mathrm{s}$ in eight parallel streams and drives the data to the correlator. The A/D and the distributor are combined on one chip to eliminate the need to run 2-Gs/s signals on the circuit board (Fig. 6). The system benefits from less noise, crosstalk, and power consumption as a result.

A possible risk in putting the distributor on the same chip as the $A / D$ is that the output signals may couple back into the analog input and distort the resulting spec- 


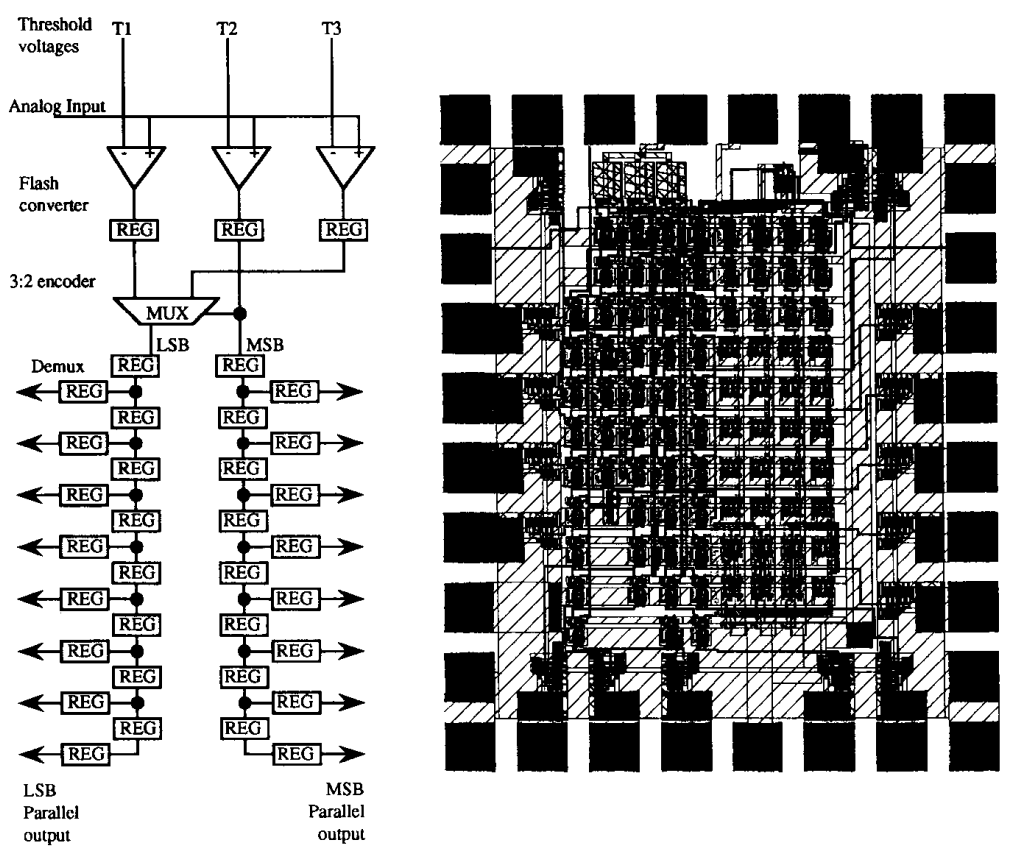

Fig. 6. Prototype GaAs 2-GHz 2-b A/D converter with a 1:8 demultiplexer/distributor.

host interface $\quad$ A/D $\quad$ correlator chips

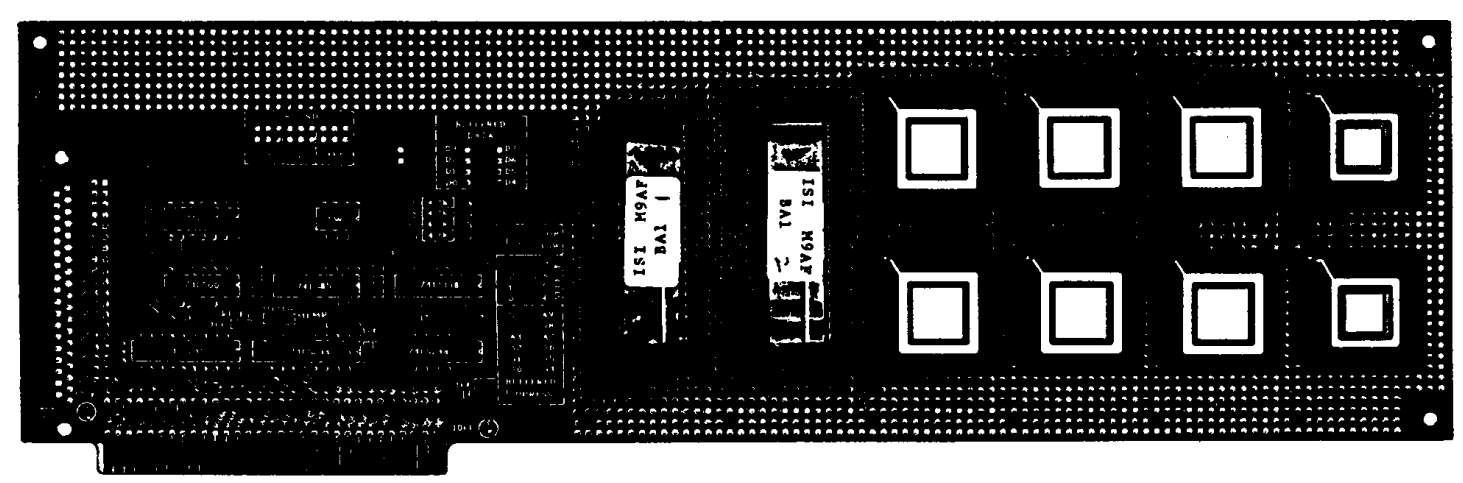

Fig. 7. Floor plan for the correlating spectrometer system on a PC-compatible circuit board.

trum in a data-dependent manner. This risk is reduced by separating analog power and ground from digital power and ground and by placing the analog and digital sections on opposite sides of the chip.

\section{System Layout}

Fig. 7 shows the layout for the digital spectrometer circuit board. The eight PGA's on the right half of the board hold the CMOS correlator chips. The two chips in the middle of the board represent the GaAs $A / D$ and demultiplexing circuitry. The connector end of the board holds the bus interface circuits. The signal and spectrometer clock are driven externally.
Correlating spectrometers for astronomy have historically required a full rack of equipment, and have been marginally reliable due to the large number of chips used. Our target implementation fits onto a single card for a PC-compatible computer, using only ten chips plus the host interface. For astronomical applications this represents a major breakthrough in cost and simplicity.

\section{Project Status}

The GaAs chips have been designed, built, and are currently undergoing testing. Preliminary CMOS test chips consisting of micropipelined delay lines and $2 \times 2-b$ multiplier circuits have been fabricated and successfully tested 
at data rates of $250 \mathrm{Ms} / \mathrm{s}$, confirming the simulations obtained with Berkeley SPICE $3 \mathrm{cl}$. The small correlator chip of Fig. 5 has been simulated, fabricated with a $2-\mu \mathrm{m}$ CMOS n-well process, and successfully tested at $50 \mathrm{Ms} / \mathrm{s}$. A new version of the chip with resized transistors is expected to operate at $250 \mathrm{Ms} / \mathrm{s}$. This chip is under fabrication; also, a large chip with eight cross-correlators is being designed.

\section{Performance Improvement}

An important figure of merit for correlating digital spectrometers is to count lags per chip times the maximum data rate of the chip, expressed in lags per second per chip. Existing designs using ECL gate arrays have achieved 2 billion and 4.4 billion lags per second per chip, not including the accumulating counters [1]. Analog CCD correlators have achieved 5 billion lags per second per chip [8] without the accumulators. The micropipelined CMOS correlator described in this paper is expected to achieve 80 billion lags per second per chip based on 320 lags and $250 \mathrm{Ms} / \mathrm{s}$. This estimate is conservative since it includes the area required for the accumulating counters.

The order-of-magnitude improvement in lags per second per chip stems from the high throughput and density available with micropipelined CMOS. The output bandwidth is reduced by integrating the correlators and the accumulators, which permits the high-density CMOS chips to run at ECL speeds. Micropipelined CMOS can dramatically boost performance when the output data rate is reduced compared to the processing data rate.

\section{ACKNOWLEDGMENT}

The author wishes to thank Prof. W. J. Dally at M.I.T. and Prof. T. G. Phillips at Caltech for reviewing the manuscript.

\section{REFERENCES}

[1] J. Wilson and K. Chandra, "Spectrometers for space microwave radiometers," Jet Propulsion Lab., Pasadena, CA, Rep., Mar. 1990.

[2] 1. E. Sutherland, "Micropiplines," Commun. $A C M$, vol. 32, no. 6, pp. 720-738, June 1989.

[3] J. Yuan and C. Svensson, "High-speed CMOS circuit technique," IEEE J. Solid-State Circuits, vol. 24, no. 1, pp. 62-70, Feb. 1989.

[4] B. F. C. Cooper, "Auto-correlation spectrometers," in Methods of Experimental Physics, vol. 12, Part B: Astrophysics and Radio Telescopes. New York: Academic, 1976, p. 280.

[5] B. F. C. Cooper, "Correlators with two-bit quantisation," Australian J. Phys., vol. 23, pp. 521-527, 1970.

[6] K. Yano et al., "A 3.8-ns CMOS $16 \times 16$-b multiplier using complementary pass-transistor logic," IEEE J. Solid-State Circuits, vol. 25, no. 2, pp. 388-395, Apr. 1990.

[7] MOSIS User Manual, Inform. Sci. Inst,, Univ. of Southern California, Marina del Rey, CA, 1988.

[8] S. C. Munroe, D. R. Arsenault, K. E. Thompson, and A. L. Lattes, "Programmable, four-channel, 128-sample, 40-Ms/s analog-ternary correlator," IEEE J. Solid-State Circuits, vol. 25, no. 2, pp. 425-429, Apr. 1990 .

\title{
A Silicon Model of an Auditory Neural Representation of Spectral Shape
}

\author{
John Lazzaro
}

\begin{abstract}
The paper describes an analog integrated circuit that implements an auditory neural representation of spectral shape. The circuit contains silicon models of the cochlea, inner hair cells, spiral ganglion cells, and the neurons that compute an amplitude-invariant representation of spectral shape. The chip uses the temporal information in each silicon auditory-nerve fiber to compute this final representation. The chip was fabricated and fully tested; the paper includes data comparing the silicon auditory-nerve representation and the final representa-
\end{abstract}

Manuscript received September 20, 1990; revised January 10, 1991 This work was supported by the Office of Naval Research and the Defense Advanced Research Projects Agency.

The author was with the California Institute of Technology, Pasadena, CA 91125 . He is now with the University of Colorado, Boulder, CO 80309-0425.

IEEE Log Number 9143199. tion. The 9000-transistor chip computes all outputs in real time using analog continuous-time processing.

\section{INTRODUCTION}

$\mathrm{T}$ HE COCHLEA is the sense organ of hearing. It converts acoustic signals into the first neural representation of audition; the auditory nerve carries this representation to the brain. Outputs from the left and right cochleas serve as inputs for the neural structures that perform spatial sound localization and sound understanding. In addition, several species of animals use their cochleas as sensors for active sonar processing.

Sound recognition, sound localization, and active sonar are practical and interesting engineering endeavors. There is renewed interest by the engineering community in 